\title{
Dispersion Corrections to the Surface Tension at Planar Surfaces
}

\author{
Linnea Lundberg and Olle Edholm* \\ Theoretical Biological Physics, Department of Theoretical Physics, \\ Royal Institute of Technology (KTH), AlbaNova University Center, \\ SE-106 91 Stockholm, Sweden \\ E-mail: oed@kth.se
}

\begin{abstract}
Molecular dynamics simulations are usually performed using cutoffs $\left(r_{c}\right)$ for the short-ranged dispersion interactions $\left(r^{-6}\right)$. For isotropic systems, long-range interactions are often added in a continuum approximation. This usually leads to excellent results that are independent of the cutoff length down to about $1 \mathrm{~nm}$. For systems with interfaces or other anisotropic systems the situation is more complicated. We study here planar interfaces, focusing on the surface tension, which is sensitive to cutoffs. Previous analytic results giving the long-range correction to the surface tension of a liquid-vapor interface as a two- or three-dimensional integral are revisited. They are generalized by introducing a dispersion density profile which makes it possible to handle multi-component systems. For the simple but common hyperbolic tangent profile the integral may be Taylor-expanded in the dimensionless parameter obtained by dividing the profile width with the cutoff length. This parameter is usually small and excellent agreement with numerical calculations of the integral is obtained by keeping two terms in the expansion. The results are compared to simulations with different lengths of the cutoff for some simple systems. The surface tension in the simulations varies linearly
\end{abstract}


in $r_{c}^{-2}$, although a small $r_{c}^{-4}$-term may be added to improve the agreement. The slope of the $r_{c}^{-2}$-line could in several cases be predicted from the change in dispersion density at the interface. The disagreements observed in some cases when comparing to theory, occur when the finite cutoff used in the simulations causes structural differences compared to long-range cutoffs or Ewald summation for the $r^{-6}$-interactions.

\section{INTRODUCTION}

When interactions are short-ranged, a cutoff is usually applied at some distance, $r_{c}$. This produces systematic errors which, however, can be corrected for. Long-range interactions, like the electrostatic ones, are in modern calculations usually handled with efficient algorithms that avoid a cutoff. When periodic boundary conditions are used, this is usually some variation of Ewald summation ${ }^{1}$ like Particle Mesh Ewald ${ }^{2}$ (PME). Although these kinds of methods may be applied for any kind of power-law potential, they have not until recently been effectively implemented for dispersion interactions in standard molecular dynamics software. ${ }^{3}$ Therefore, it is still of interest to calculate long-range corrections. The cutoff affects energies, forces and pressures. The surface tension is therefore also affected since it may be obtained as ${ }^{4}$

$$
\gamma=\int\left(p_{\mathrm{n}}-p_{\mathrm{l}}(z)\right) \mathrm{d} z
$$

for a surface that is oriented with the normal in the $z$-direction. $p_{\mathrm{n}}$ is the normal pressure and $p_{1}(z)$ is the lateral $(\mathrm{x}-\mathrm{y})$ pressure. The short-range interactions are here modeled by a Lennard-Jones potential

$$
U_{L J}(r)=4 \varepsilon\left(\left(\frac{\sigma}{r}\right)^{12}-\left(\frac{\sigma}{r}\right)^{6}\right)=\frac{C_{12}}{r^{12}}-\frac{C_{6}}{r^{6}}
$$

where $r$ is the distance between two particles, $\epsilon$ the strength of the interaction and $\sigma$ the distance at which the potential goes from being repulsive to being attractive. We consider here long-range corrections to the dispersive $r^{-6}$-interactions. For the $r^{-12}$-interaction a 
cutoff (without any corrections) is usually accurate enough, although similar long-range corrections could be calculated for that interaction.

The long-range dispersion corrections can often be neglected for energy and pressures. For the surface tension, the long-range effects from the seemingly weak and rapidly decaying van der Waals interactions are, on the contrary, of crucial importance. For a Lennard-Jones fluid, the usage of a standard cutoff of $2.25 \sigma$ results in a surface tension that is only about one third of the experimental value (or the value obtained using much larger simulation cutoffs).

Chapela et al. ${ }^{5}$ derived our Eqn. 15 in 1977 as a cutoff correction to the surface tension. Their equation for the special case of a hyperbolic tangent profile was later corrected by Blokhuis et al. ${ }^{6}$ in 1995 to give our Eqn. 19. These expressions have been widely used since then. Recently Neyt et al. ${ }^{7}$ applied numerical long-range corrections to the surface tension and obtained quantitative predictions of the interfacial tensions at liquid-liquid interfaces using atomistic as well as coarse-grained models. Their work shows that long-range dispersion corrections to the $r^{-6}$-interactions are necessary to obtain reliable results.

In this work we generalize previous results and derive an expression for the tail-correction to the surface tension for multi-component systems. Without tail-correction the surface tension calculated from the simulations depends on the cutoff, but when a proper $r_{c}^{-2}$-correction to the surface tension is added it becomes independent on the cutoff. The resulting surface tension obtained after applying a long-range correction is also closer to the experimental value for the systems studied here. We also derive similar tail-corrections to energy and average pressures although these are of less importance.

The systems studied in the simulations are cyclohexane/vacuum, water/vacuum, water/cyclohexane and membrane/water, with cyclohexane modeled as a Lennard-Jones fluid. Cyclohexane was chosen because we wanted a molecule without electrostatic interactions that is liquid at similar temperatures as water. This makes it possible to study a simple model for an interface between water and a hydrophobic solvent. The 'vacuum' parts of the 
first two simulated systems consist after equilibration of vapor. All values and expressions for the surface tension are in this paper given for one surface, although the simulated systems contain two surfaces due to the periodic boundaries employed.

In this paper we will first present the theory and the theoretical results before we turn to the simulations and the verification of the theory.

\section{THEORY}

The pressure, $p$, is calculated from the virial theorem in simulations as

$$
p=\frac{N k_{B} T}{V}+\frac{1}{6 V} \sum_{i=1}^{N} \sum_{j=1}^{N}\left\langle\mathbf{F}_{i j} \cdot \mathbf{r}_{i j}\right\rangle
$$

where the sum goes over all $N$ particles in the system and pairwise interactions are assumed. The van der Waals energy between two interacting particles at distance $r_{i j}$ is

$$
E_{i j}\left(r_{i j}\right)=-\frac{C_{6}^{i j}}{r_{i j}^{6}}
$$

resulting in the force

$$
\mathbf{F}_{i j}=-\nabla E_{i j}=-\frac{6 C_{6}^{i j}}{r_{i j}^{8}} \mathbf{r}_{i j}
$$

For a single component system, the correction to the pressure from distances outside the cutoff can be written in terms of the number density $\rho_{V}(\mathbf{r})$ as

$$
p^{\text {tail }}=-\frac{N}{V} C_{6} \int_{r \geq r_{c}} \frac{\rho_{V}(\mathbf{r})}{r^{6}} \mathrm{~d}^{3} r,
$$

while the corresponding correction to the energy is

$$
E^{\text {tail }}=-\frac{N}{2} C_{6} \int_{r \geq r_{c}} \frac{\rho_{V}(\mathbf{r})}{r^{6}} \mathrm{~d}^{3} r .
$$


For constant $\rho_{V}(\mathbf{r})=N / V$, the integrals can be performed to obtain

$$
p^{\text {tail }}=-\frac{4 \pi C_{6}}{3 r_{c}^{3}}\left(\frac{N}{V}\right)^{2}
$$

and

$$
E^{\text {tail }}=-\frac{2 \pi C_{6}}{3 r_{c}^{3}} \frac{N^{2}}{V}
$$

See for instance Allen and Tildesley. ${ }^{8}$

If these corrections are added, simulations give the same result independent of cutoff for isotropic and homogeneous systems (above some minimum cutoff below which the structure of the systems is affected). For anisotropic, inhomogeneous systems containing different kinds of atoms, the situation is more complicated, but similar general correction formulas can be derived. When the $C_{6}$-parameters between different atoms can be written as geometrical averages we have

$$
E_{i j}\left(r_{i j}\right)=-\frac{C_{6}^{i j}}{r_{i j}^{6}}=-\frac{\sqrt{C_{6}^{i} C_{6}^{j}}}{r_{i j}^{6}}=-4 \sqrt{\epsilon_{i} \epsilon_{j}} \frac{\sigma_{i}^{3} \sigma_{j}^{3}}{r_{i j}^{6}} .
$$

Usually the $\epsilon$-parameters are treated as geometrical averages, but for the $\sigma$-parameters arithmetic or geometric averages may be used. We stick here to a treatment with geometric averages for both. Then we may introduce a density of the square root of the $C_{6}$ parameter (dispersion density) as

$$
\rho(\mathbf{r})=\sum_{c} \sqrt{C_{6}^{c}} \rho_{V}^{c}(\mathbf{r})=\sum_{i} \sqrt{C_{6}^{i}} \rho_{V}^{i}(\mathbf{r})
$$

were $c$ denotes the component (atom) type, $\rho_{V}^{c}$ is the number density of component $c$ and $i$ denotes a single atom. The dimension of $\rho$ is square root of energy. Then the tail correction 
to the diagonal components of the averaged pressure tensor, $\left\langle p_{\alpha \alpha}\right\rangle$ may then be written as

$$
\begin{aligned}
& \left\langle p_{\alpha \alpha}^{\text {tail }}\right\rangle \\
& =-\frac{1}{V} \int_{\left|\mathbf{r}_{1}-\mathbf{r}_{2}\right| \geq r_{c}} \frac{\left(\mathbf{r}_{1}-\mathbf{r}_{\mathbf{2}}\right)_{\alpha}^{2}}{\left|\mathbf{r}_{1}-\mathbf{r}_{2}\right|^{8}} \rho\left(\mathbf{r}_{1}\right) \rho\left(\mathbf{r}_{2}\right) \mathrm{d}^{3} r_{1} \mathrm{~d}^{3} r_{2},
\end{aligned}
$$

with $\alpha$ being $x, y$ or $z$. This 6 -dimensional integral cannot be analytically solved in general but there are interesting special cases that may be treated. In planar geometry, the density is a function of one single variable which we take as $z$. Then the tail-corrections to the average pressures may be rewritten as 3-dimensional integrals

$$
\begin{aligned}
\left\langle p_{n}^{\text {tail }}\right\rangle & =\left\langle p_{z z}^{\text {tail }}\right\rangle \\
& =\frac{-6 \pi}{2 L} \int_{-L}^{L} \int_{-1}^{1} \int_{r_{c}}^{\infty} \frac{s^{2} \rho(z) \rho(z-r s)}{r^{4}} \mathrm{~d} r \mathrm{~d} s \mathrm{~d} z
\end{aligned}
$$

and

$$
\begin{aligned}
& \left\langle p_{l}^{\text {tail }}\right\rangle=\left\langle p_{x x}^{\text {tail }}\right\rangle=\left\langle p_{y y}^{\text {tail }}\right\rangle \\
& \quad=\frac{-3 \pi}{2 L} \int_{-L}^{L} \int_{-1}^{1} \int_{r_{c}}^{\infty} \frac{\left(1-s^{2}\right) \rho(z) \rho(z-r s)}{r^{4}} \mathrm{~d} r \mathrm{~d} s \mathrm{~d} z
\end{aligned}
$$

where $s=\left(z_{1}-z_{2}\right) / r, r$ the distance between two particles with $z$-coordinates $z_{1}$ and $z_{2}$, (here as $z_{1}=z$ and $z_{2}=z-r s$ ) and $2 L$ is the extension of the system in the normal direction. $p_{n}$ and $p_{l}$ are the pressures in the normal and lateral directions. Note that the tail-pressures all reduce to Eqn. 8 if we insert a constant $\rho=\sqrt{C_{6}} N / V$ and perform the integrations. The corrections to the pressure components are all negative (since all integrands are positive). The tail correction to the surface tension may now be expressed as

$$
\begin{aligned}
\gamma^{\text {tail }} & =\int\left[p_{n}^{\text {tail }}-p_{l}^{\text {tail }}\right] \mathrm{d} z \\
& =3 \pi \int_{-L}^{L} \int_{-1}^{1} \int_{r_{c}}^{\infty} \frac{1-3 s^{2}}{r^{4}} \rho(z) \rho(z-r s) \mathrm{d} r \mathrm{~d} s \mathrm{~d} z
\end{aligned}
$$


Note here that a constant $\rho$ makes it possible to do the $s$-integral directly to obtain the tail surface tension zero.

In the same way we get the tail contribution to the attractive Lennard-Jones energy in the general case

$$
E^{\text {tail }}=-\frac{1}{2} \int_{\left|\mathbf{r}_{1}-\mathbf{r}_{2}\right| \geq r_{c}} \frac{\rho\left(\mathbf{r}_{1}\right) \rho\left(\mathbf{r}_{2}\right)}{\left|\mathbf{r}_{1}-\mathbf{r}_{2}\right|^{6}} \mathrm{~d}^{3} r_{1} \mathrm{~d}^{3} r_{2}
$$

In planar geometry, this gives the energy per volume

$$
\frac{E^{\text {tail }}}{V}=-\frac{\pi}{2 L} \int_{-L}^{L} \int_{r_{c}}^{\infty} \int_{-1}^{1} \frac{\rho(z) \rho(z-r s)}{r^{4}} \mathrm{~d} s \mathrm{~d} r \mathrm{~d} z,
$$

with $V$ being the volume of the system. We note that this reduces to Eqn. 9 for an isotropic system where $\rho=\sqrt{C_{6}} N / V$ is constant.

\subsection{A Hyperbolic Tangent-Shaped Interface}

We now turn to a special case, a realistic density profile that can be justified theoretically in some cases and also often is quite well reproduced by simulations, the hyperbolic tangent profile (see e.g. Allen and Tildesley ${ }^{8}$ or Safran ${ }^{9}$ )

$$
\rho(z)=\rho_{0}+\frac{1}{2} \Delta \rho \tanh (z / d)
$$

Here $\rho_{0}$ is the average attractive dispersion density, $\Delta \rho$ the dispersion density difference between the two regions, while the parameter $d$ describes the extension of the interface region. The special case, $d=0$, gives a Heaviside function profile which also is of interest. The tail-correction to the surface tension may then be written as

$$
\gamma^{\text {tail }}=3 \pi(\Delta \rho)^{2} \int_{0}^{1} \int_{r_{c}}^{\infty} \frac{\left(3 s^{3}-s\right) \operatorname{coth}(s r / d)}{r^{3}} \mathrm{~d} r \mathrm{~d} s
$$


This is a slight generalization of the result in Blockhuis et al. ${ }^{6}$ to which Eqn. 19 reduces for a single component liquid-vapor system that has $\Delta \rho=\sqrt{C_{6}} \Delta \rho=\sqrt{C_{6}}\left(\rho_{l}-\rho_{v}\right) \approx \sqrt{C_{6}} \rho_{l}$, with the number densities $\rho_{l}$ and $\rho_{l}$ for the liquid and the vapor. The integral cannot be solved analytically but the equation may be rewritten as

$$
\gamma^{\text {tail }}=\frac{3 \pi(\Delta \rho)^{2}}{8 r_{c}^{2}} f_{\gamma}\left(d / r_{c}\right)
$$

where $f_{\gamma}\left(d / r_{c}\right)$, which contains the $d$-dependence and is independent of $\Delta \rho$, is

$$
f_{\gamma}\left(d / r_{c}\right)=\int_{0}^{1} \int_{0}^{1} 8 x\left(3 s^{3}-s\right) \operatorname{coth}\left(s r_{c} / x d\right) \mathrm{d} x \mathrm{~d} s
$$

It is easily shown that $f_{\gamma}\left(d / r_{c}\right)$ is 1 for $d / r_{c}=0$, corresponding to the Heaviside function profile. The integral, which is not analytically solvable, can be numerically calculated but may also be Taylor-expanded in the usually small parameter $d / r_{c}$. The agreement between the expansion and the numerical result is excellent with terms up to fourth order (see Fig. 1)

$$
\gamma^{\text {tail }}=\frac{3 \pi(\Delta \rho)^{2}}{8 r_{c}^{2}}\left[1-\frac{\pi^{2}}{6}\left(\frac{d}{r_{c}}\right)^{2}+3.53\left(\frac{d}{r_{c}}\right)^{4}+\ldots\right]
$$

Similar corrections can be calculated for the energy and the separate pressure components. We obtain for the energy

$$
\begin{aligned}
& \frac{E^{\text {tail }}}{V} \\
& =-\frac{2 \pi}{3 r_{c}^{3}}\left(\rho_{0}^{2}+\left(\frac{\Delta \rho}{2}\right)^{2}\right)+\frac{\pi}{16} \frac{(\Delta \rho)^{2}}{r_{c}^{3}} \frac{r_{c}}{2 L} f_{E}\left(d / r_{c}\right) .
\end{aligned}
$$

Note that when the linear dimension of the system in the normal direction is much larger than $r_{c}$, the second term is negligible. The first (bulk) term is just the average of the correction for two systems with the limiting densities. In the general case when the two regions are of 


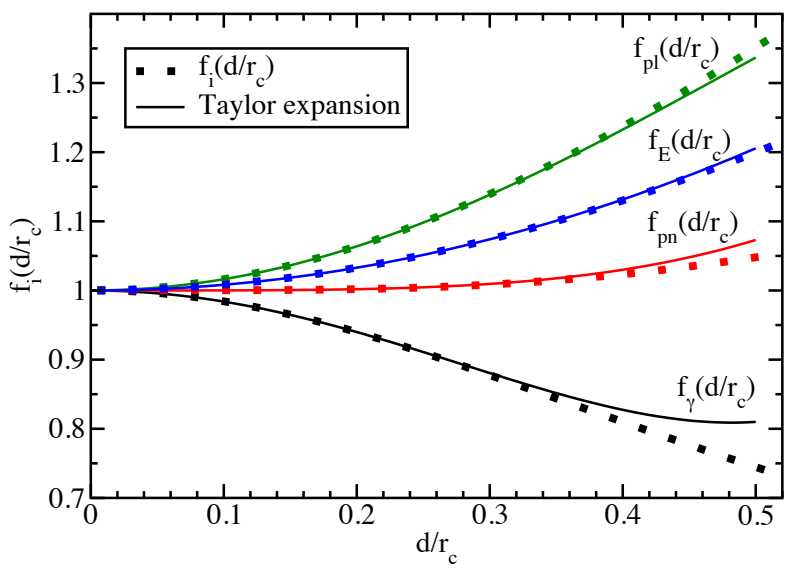

Figure 1: The functions $f_{i}\left(d / r_{c}\right)(\mathrm{i}=\gamma, E, p n, p l)$ describe the dependency of surface tension, energy, normal and lateral pressures upon the dimensionless parameter $d / r_{c}$ for a tanhsurface profile. Note that all functions become 1 for $d=0$ (Heaviside function) or when the cutoff approaches infinity. The solid lines represent Taylor expansions up to fourth order (see Eqns. 22 and 30-32).

unequal size the bulk term is

$$
-\frac{2 \pi}{3 r_{c}^{3}}\left\langle\rho^{2}\right\rangle=-\frac{2 \pi}{3 r_{c}^{3}}\left(\alpha \rho_{1}^{2}+(1-\alpha) \rho_{2}^{2}\right)
$$

with $0 \leq \alpha \leq 1$ and $\rho_{i}$ being the dispersion density in region $i$. The dependence of the extension of the interface is given by the function

$$
f_{E}\left(d / r_{c}\right)=\int_{0}^{1} \int_{0}^{1} 4 x s \operatorname{coth}\left(s r_{c} / x d\right) \mathrm{d} x \mathrm{~d} s
$$

which also is 1 in the limit $d / r_{c}=0$. In the same way, the tail-corrections to the average normal and lateral pressures are obtained as

$$
\left\langle p_{n}^{\text {tail }}\right\rangle=-\frac{4 \pi}{3 r_{c}^{3}}\left\langle\rho^{2}\right\rangle+\frac{3 \pi}{4} \frac{(\Delta \rho)^{2}}{r_{c}^{3}} \frac{r_{c}}{2 L} f_{p n}\left(d / r_{c}\right)
$$

with

$$
f_{p n}\left(d / r_{c}\right)=\int_{0}^{1} \int_{0}^{1} 8 x s^{3} \operatorname{coth}\left(s r_{c} / x d\right) \mathrm{d} x \mathrm{~d} s
$$


and

$$
\left\langle p_{l}^{\text {tail }}\right\rangle=-\frac{4 \pi}{3 r_{c}^{3}}\left\langle\rho^{2}\right\rangle+\frac{3 \pi}{8} \frac{(\Delta \rho)^{2}}{r_{c}^{3}} \frac{r_{c}}{2 L} f_{p l}\left(d / r_{c}\right),
$$

with

$$
f_{p l}\left(d / r_{c}\right)=\int_{0}^{1} \int_{0}^{1} 8 x\left(s-s^{3}\right) \operatorname{coth}\left(s r_{c} / x d\right) \mathrm{d} x \mathrm{~d} s
$$

Both these functions are 1 in the limit $d / r_{c}=0$ and can be expanded in $d / r_{c}$ as shown in Fig. 1.

To summarize, we may write the tail-corrections for energy and pressures as Taylor expansions

$$
\begin{aligned}
& \frac{E^{\text {tail }}}{V}=-\frac{2 \pi}{3 r_{c}^{3}}\left\langle\rho^{2}\right\rangle+ \\
& \frac{\pi}{16} \frac{(\Delta \rho)^{2}}{r_{c}^{3}} \frac{r_{c}}{2 L}\left[1+\frac{\pi^{2}}{12}\left(\frac{d}{r_{c}}\right)^{2}+\ldots\right], \\
&\left\langle p_{n}^{\text {tail }}\right\rangle=-\frac{4 \pi}{3 r_{c}^{3}}\left\langle\rho^{2}\right\rangle+ \\
& \frac{3 \pi}{4} \frac{(\Delta \rho)^{2}}{r_{c}^{3}} \frac{r_{c}}{2 L}\left[1+1.17\left(\frac{d}{r_{c}}\right)^{4}+\ldots\right]
\end{aligned}
$$

and

$$
\begin{aligned}
& \left\langle p_{l}^{\text {tail }}\right\rangle=-\frac{4 \pi}{3 r_{c}^{3}}\left\langle\rho^{2}\right\rangle+ \\
& \frac{3 \pi}{8} \frac{(\Delta \rho)^{2}}{r_{c}^{3}} \frac{r_{c}}{2 L}\left[1+\frac{\pi^{2}}{6}\left(\frac{d}{r_{c}}\right)^{2}-1.19\left(\frac{d}{r_{c}}\right)^{4}+\ldots\right] .
\end{aligned}
$$

These expressions consist of a bulk term and a surface term where the bulk term usually dominates. There are analytic expressions for the numerical coefficients but these are lengthy. For the surface tension, which is obtained as the integral over the difference between the normal and lateral pressures, the bulk terms disappear and we are left with a leading interface term in Eqn. 22. If the system has a sharp interface $\left(d=0\right.$ or $\left.d / r_{c} \ll 1\right)$ the expressions simplify and we are left with a $r_{c}^{-2}$-term in the tail-correction. 


\section{SIMULATIONS}

All simulations were performed using GROMACS 5.0. ${ }^{10-12}$ The water model was TIP4P $/ 2005^{13}$ and the membrane model was Dipalmitoylphosphatidylcholine (DPPC). ${ }^{14}$ The LennardJones parameters of the single particle model for cyclohexane were determined to give agreement with the bulk density $\left(773.9 \mathrm{~kg} / \mathrm{m}^{3}\right)$ and the heat of vaporization $(33.01 \mathrm{~kJ} / \mathrm{mol})$ of cyclohexane $^{15}$ at $300 \mathrm{~K}$ using Lennard-Jones PME (LJ-PME) ${ }^{3}$ or isotropic cutoff corrections.

Table 1: The Lennard-Jones parameters for the different components. The values for the membrane are averages over all the different lipid atoms.

\begin{tabular}{lccc}
\hline Parameter & Cyclohexane & Water $^{13}$ & Membrane $^{14}$ \\
\hline$\sigma[\mathrm{nm}]$ & 0.56180 & 0.31589 & 0.38171 \\
$\epsilon[\mathrm{kJ} / \mathrm{mol}]$ & 4.08650 & 0.7749 & 0.16348 \\
$\mathrm{C}_{6}\left[\mathrm{~J} / \mathrm{mol} \mathrm{nm}{ }^{6}\right]$ & 513.90 & 3.0798 & 6.3554 \\
$\mathrm{C}_{12}\left[\mathrm{~mJ} / \mathrm{mol} \mathrm{nm}^{12}\right]$ & 16.157 & 3.060 & 24.722
\end{tabular}

The Lennard-Jones parameters used in the simulations are shown in Table 1. The lengths of the simulations were different for the different systems, but they were enough to make sure that surface tension and energies were stable and satisfactory statistical errors could be estimated. Typically this meant 10-50 ns. The dispersion density $(\rho)$ is varying with $z$ and this is shown in Fig. 2 as calculated from the simulations (using LJ-PME).

The simulations with a vacuum part were started from bulk NpT-simulations in which the periodic box was elongated by a factor $2-9$ in one direction $(z)$, after which the simulations were continued in an NVT-ensemble. In principle, these simulations could be seen as simulations of a liquid-gas interface. The small number of molecules and the weak interactions in the gas makes it difficult to obtain statistically accurate results for the gas phase. Therefore we did not find it suitable to run these systems in an NpT-ensemble. The two-component systems were simulated at a pressure of 1 bar in the normal direction to the surface (Parrinello-Rahman scaling ${ }^{16}$ ) and in a fixed box in the two lateral directions. The sizes of the different systems along with the fractions of the different compounds are shown in 


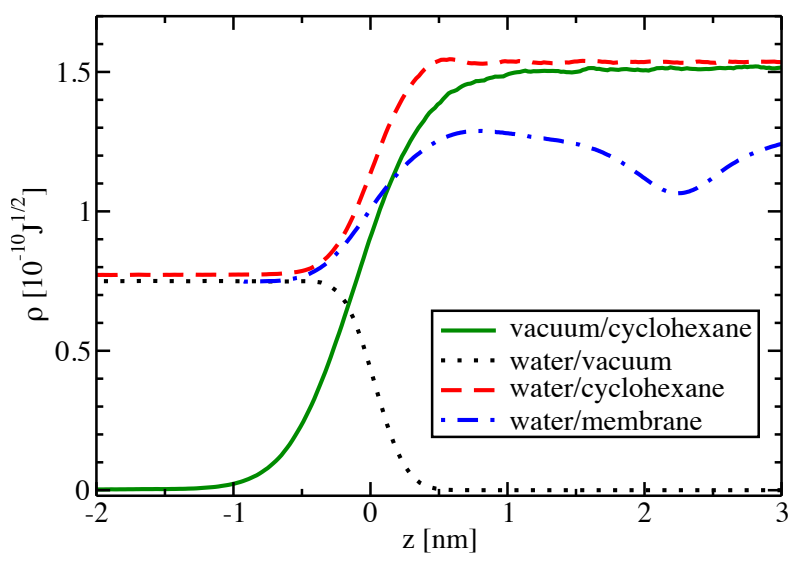

Figure 2: The dispersion density, $\rho(z)$, for the different systems with the interfaces located at $z=0$, as calculated from simulations using LJ-PME.

Table 2. All simulations were performed at $300 \mathrm{~K}$, except the membrane simulations which were performed at $323 \mathrm{~K}$. To maintain the temperature V-rescale ${ }^{17}$ was used. We performed the simulations using cutoffs from 1 to $4 \mathrm{~nm}$ or with LJ-PME, which corresponds to infinite cutoff.

Table 2: System parameters. The volume (V) and length in the normal direction (2L) of the systems including the vacuum part. The area (A) is a square in the lateral (xy) plane. $\mathrm{V}_{n}$ is the volume of the two parts of the system in percent and $\mathrm{N}_{n}$ is the number of molecules of each component.

\begin{tabular}{lcccccc}
\hline System $(1 / 2)$ & $\mathrm{V}\left[\mathrm{nm}^{3}\right]$ & $2 \mathrm{~L}[\mathrm{~nm}]$ & $\mathrm{A}\left[\mathrm{nm}^{2}\right]$ & $\mathrm{V}_{1} / \mathrm{V}_{2}[\%]$ & $\mathrm{N}_{1}$ & $\mathrm{~N}_{2}$ \\
\hline $\mathrm{CH} /$ vacuum & 1966.27 & 20.0 & 98.31 & $50 / 50$ & 5331 & 0 \\
Water/vacuum & 2160 & 60.0 & 36 & $10 / 90$ & 7100 & 0 \\
$\mathrm{CH} /$ water & 1997.90 & 20.3 & 98.42 & $50 / 50$ & 5331 & 32587 \\
DPPC/water & 1152.46 & 7.0 & 164.64 & $53 / 47$ & 512 & 18228
\end{tabular}

\section{RESULTS AND DISCUSSION}

Here we use simulations of the four different systems described above to test the theory. The variation in surface tension with cutoff is compared to theory. Table 3 shows the parameters used to calculate the corrections from Eqn. 22.

For cyclohexane modeled as a Lennard-Jones solvent, the results are shown in Fig. 3. 
Table 3: The values of the dispersion density difference, $(\Delta \rho)^{2}$, and the surface thicknesses, $d$, calculated from a least square fit of Eqn. 18 to the dispersion densities in Fig. 2. $a$ and $b$ are the two first coefficients in a Taylor expansion of the surface tension, $\gamma\left(r_{c}\right)=\gamma_{\infty}+a / r_{c}^{2}+b / r_{c}^{4}$, where $a=-3 \pi(\Delta \rho)^{2} / 8$ and $b=\pi^{3}(d \Delta \rho)^{2} / 16$.

\begin{tabular}{lcccc}
\hline System & $(\Delta \rho)^{2} \times 10^{20}[\mathrm{~J}]$ & $d[\mathrm{~nm}]$ & $a \times 10^{21}[\mathrm{Nm}]$ & $b \times 10^{39}\left[\mathrm{Nm}^{3}\right]$ \\
\hline CH/vacuum & 2.307 & 0.50 & -27.18 & 11.18 \\
Water/vacuum & 0.562 & 0.21 & -6.62 & 0.48 \\
CH/water & 0.582 & 0.27 & -6.86 & 0.82 \\
DPPC/water & 0.291 & 0.38 & -3.43 & 0.81
\end{tabular}

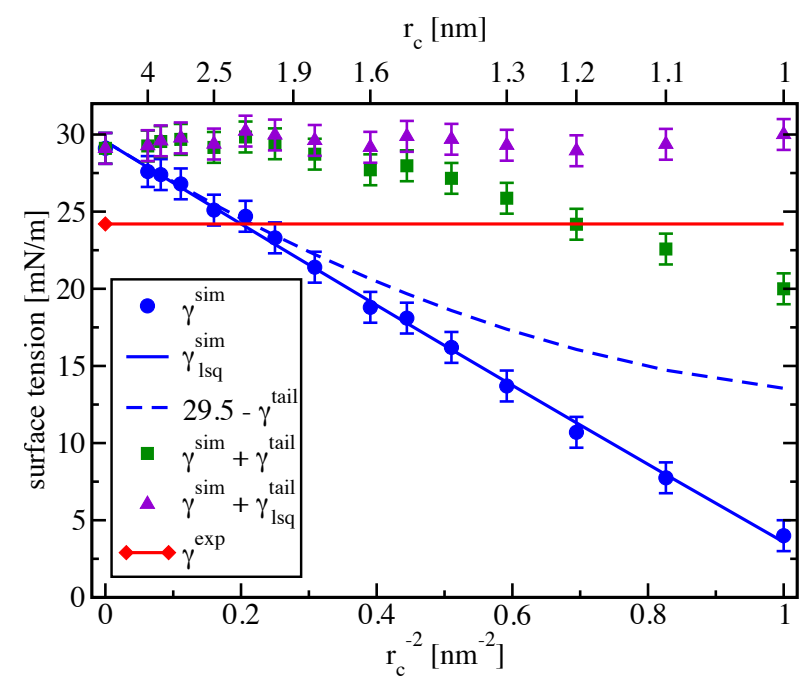

Figure 3: Surface tension of a cyclohexane/vacuum surface at $300 \mathrm{~K}$. The experimental value of the surface tension is $24.2 \mathrm{mN} / \mathrm{m}$ at $300 \mathrm{~K} .{ }^{18}$ The tail-correction is $\gamma^{\text {tail }}=27.18 / r_{c}^{2}-$ $11.18 / r_{c}^{4}$ (see Table 3) while a least square fit of the simulation data is $\gamma_{\mathrm{lsq}}^{\mathrm{sim}}=29.5-26.9 / r_{c}^{2}+$ $1.0 / r_{c}^{4}(\mathrm{mN} / \mathrm{m})$, where the $\gamma_{\mathrm{lsq}}^{\text {tail }}$ is the last two terms of the fit.

It is clear from the figure that simulations result in surface tensions from 3.5 to $30 \mathrm{mN} / \mathrm{m}$ depending on the cutoff. Applying isotropic dispersion corrections does not change anything since these corrections are the same for the normal and lateral pressures. With the anisotropic corrections from Table 3 applied, we obtain a surface tension between 20 and $30 \mathrm{mN} / \mathrm{m}$ depending on the cutoff (going from 1 to $4 \mathrm{~nm}$ ). The difference compared to the experimental surface tension of $24.2 \mathrm{mN} / \mathrm{m}$ is most likely due to the fact that the Lennard-Jones fluid model of cyclohexane does not catch all properties of this fluid perfectly.

The simulation data fits $\gamma_{\infty}+a / r_{c}^{2}+b / r_{c}^{4}$ (see Table 3$)$, where the $r_{c}^{-4}$-term is small $\left(\gamma_{\infty}\right.$ 


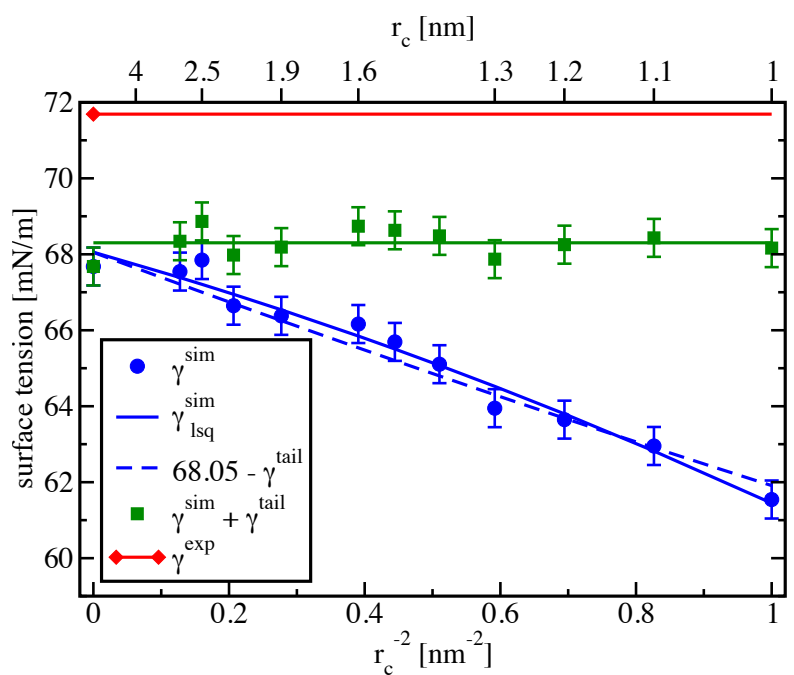

Figure 4: Surface tension of a water/vacuum surface at $300 \mathrm{~K}$. The experimental value of the surface tension is $71.69 \mathrm{mN} / \mathrm{m}$ at $300 \mathrm{~K} .{ }^{21}$ The tail-correction is $\gamma^{\text {tail }}=6.62 / r_{c}^{2}-0.48 / r_{c}^{4}$ (see Table 3), while a least square fit of the simulation data is $\gamma_{\mathrm{lsq}}^{\mathrm{sim}}=68.05-5.0 / r_{c}^{2}-1.6 / r_{c}^{4}$ $(\mathrm{mN} / \mathrm{m})$.

is the surface tension at infinite cutoff). The theory gives the coefficient $a$ but a relatively large negative $b$, which does not agree with the small positive value obtained in the least square fit. This could be explained from density deviations in the system simulated with small cutoffs, which was also observed by Nijmeijer et al. ${ }^{19}$

The present data can be transformed into dimensionless (Lennard-Jones) units and back to physical units of other systems. For liquid argon, the present simulations would then correspond to a surface tension of about $12 \mathrm{mN} / \mathrm{m}$ (for infinite cutoff) in good agreement with experiment $(13 \mathrm{mN} / \mathrm{m}$ at $85 \mathrm{~K}) .{ }^{20}$

For the TIP4P/2005 water surface, the agreement between theory (with the parameters taken from Table 3) and simulation is almost perfect (see Fig. 4). The difference between the surface tension with anisotropic corrections and the experimental value is $4 \mathrm{mN} / \mathrm{m}(5 \%)$, which is less than for other water models but still not perfect, as also reported by Vega and de Miguel. ${ }^{22}$

For the cyclohexane/water surface the simulated data follows a $r_{c}^{-2}$ line, as can be seen in Fig. 5. There is a slight difference in slope between the theoretical curve (data from 


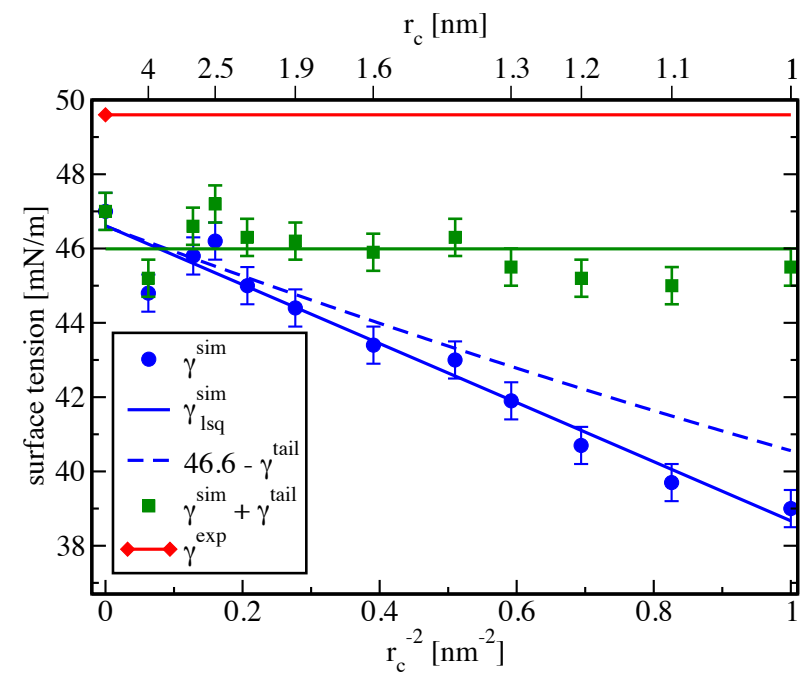

Figure 5: Surface tension of a water/cyclohexane surface at $300 \mathrm{~K}$. The experimental value is $49.6 \mathrm{mN} / \mathrm{m}$ at $298 \mathrm{~K}^{23}$ The tail-correction to the surface tension is $\gamma^{\text {tail }}=6.86 / r_{c}^{2}-0.82 / r_{c}^{4}$ (see Table 3) while the least square fit tail-correction is $\gamma_{\mathrm{lsq}}^{\mathrm{sim}}=46.6-7.9 / r_{c}^{2}-0.02 / r_{c}^{4}$ $(\mathrm{mN} / \mathrm{m})$.

Table 3) and the simulations, but the difference is significantly smaller than in the cyclohexane/vacuum case.

The deviations between experimental and calculated surface tensions (infinite cutoff) are $5 \%$ for the water, $6 \%$ for the water/cyclohexane-surface and $18 \%$ for the cyclohexane/vacuum surface. These differences reflect small deficiencies in the underlying atomistic models.

For the membrane, the variation of surface tension with the cutoff follows again a straight line versus $r_{c}^{-2}$ but it is about $50 \%$ steeper than predicted from the parameters in Table 3, as can be seen in Fig. 6. There are some clear problems in the membrane case. First, there is a deviation from the tanh-profile most clearly reflected in the dip of density in the middle of the membrane (see Fig. 2). Secondly, the membrane thickness is small, although still larger than the cutoffs. Thirdly, the membrane system is repeated periodically in the $z$-direction every $7 \mathrm{~nm}$ which is less than the periodic box of the other systems (20-60 nm) but still longer, although not much longer, than the cutoffs. All of this can be corrected for by calculating the tail-corrections numerically including periodic copies of the system. For numerically calculating the correction, the more general Eqn. 15 and the dispersion densities of Fig. 2 


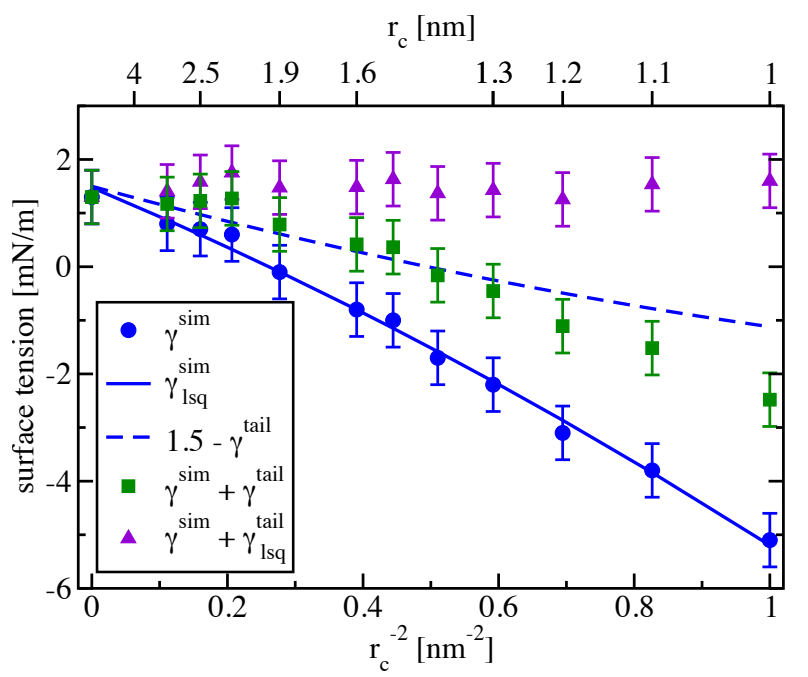

Figure 6: Surface tension of a membrane/water surface at $323 \mathrm{~K}$. The tail-correction to the surface tension is $\gamma^{\text {tail }}=2.43 / r_{c}^{2}-0.81 / r_{c}^{4}$ (see Table 3) while the least square fit of the simulation data is $\gamma_{\mathrm{lsq}}^{\mathrm{tail}}=1.5-5.3 / r_{c}^{2}-1.4 / r_{c}^{4}$.

were used. This does, however, not change the results much. We have also added more water in the simulations without achieving any improved agreement with theory. When the corrections have been calculated in these different ways the results have differed less than $5 \%$ from each other. We also observe some small differences in the lipid number density depending on the cutoff, but these are not large enough to explain the different slopes in Fig. 6.

In the water and cyclohexane systems the surface tension is an experimentally accessible material property of the fluid (or fluid mixtures). For membranes, the corresponding quantity is the area per lipid. At the equilibrium area the free energy of the system is minimal and hence the surface tension is zero $\left(\gamma=\left(\frac{\partial G}{\partial A}\right)_{T, p}=0\right)$. The present simulations were performed at the fixed area per lipid $0.643 \mathrm{~nm}^{2}$ and showed (without dispersion corrections) a surface tension per bilayer between -10 and $3 \mathrm{mN} / \mathrm{m}$ for cutoffs between $1 \mathrm{~nm}$ and infinity (LJPME). It is possible to calculate the change in area per lipid necessary to give zero surface tension since $\Delta A=\frac{\Delta \gamma}{K_{A}} A$, where $K_{A}$ is the area compressibility modulus of the membrane, which in this case is about $300 \mathrm{mN} / \mathrm{m} .{ }^{24}$ This would give the area per lipid from $0.638 \mathrm{~nm}^{2}$ (LJ-PME) up to $0.665 \mathrm{~nm}^{2}\left(r_{c}=1 \mathrm{~nm}\right)$ at zero surface tension. The experimental value ${ }^{25}$ 
is $0.630 \pm 0.010 \mathrm{~nm}^{2}$. The area per lipid with LJ-PME is within experimental uncertainty, while the value obtained from the simulation with the shortest cutoff clearly falls outside the experimental error bars. The systematic error $0.027 \mathrm{~nm}^{2}$ resulting from the use of a $1 \mathrm{~nm}$ cutoff could be reduced to below $0.01 \mathrm{~nm}^{2}$ (the experimental error) either by increasing the cutoff to at least $1.6 \mathrm{~nm}$ or by combining a smaller cutoff of $1.3 \mathrm{~nm}$ with the application of anisotropic dispersion corrections.

Common for Figs. 3-6 is that the simulations give a too small surface tension at the shortest cutoffs. This may be corrected with an expression $a / r_{c}^{2}+b / r_{c}^{4}$, where the first term is the important one. In several cases the coefficient of the first term is excellently predicted from the dispersion density discontinuity at the interface.

The density profiles of the membrane and of the water show only a weak dependence upon the cutoff. For the Lennard-Jones solvent (cyclohexane) the difference is more important. We note first that even with LJ-PME for the dispersion interactions, there is a drop in the number density for a system with a surface compared to a bulk simulation. In the cyclohexane/vacuum system it is around $8 \%$ and in the cyclohexane/water system about $1.5 \%$. In the cyclohexane/vacuum system there is a large further drop in the number density at the shortest cutoffs. With a $1 \mathrm{~nm}$ cutoff, this drop is $16 \%$ (compared to the LJ-PME simulation) and we also obtain some dense gas phase in equilibrium with the fluid. The reason for this is that with a shorter cutoff the effective attraction becomes weaker, corresponding to a higher temperature which brings the system closer to the critical point.

The tail-corrections for the energy, $E^{\text {tail }}$, are calculated using Eqn. 17 and the resulting corrections are shown in Fig. 7. The simulated energy of the systems is dependent on the cutoff and is linear in $r_{c}^{-3}$. The surface term proportional to $r_{c}^{-2}$ is not distinguishable with the accuracy of the simulations. When the corrections are added the energy becomes independent of the cutoff.

The corrections to the pressures (Eqns. 31 and 32) range from about 400 atm at $r_{c}=1 \mathrm{~nm}$ to about $10 \mathrm{~atm}$ at $r_{c}=3 \mathrm{~nm}$. These are large absolute values but since condensed matter 


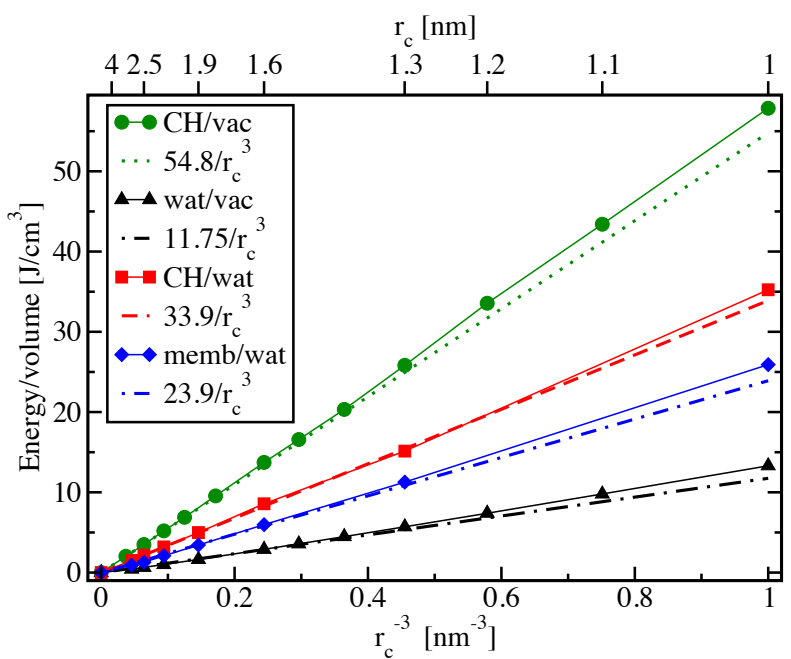

Figure 7: The energy corrections per volume, where the volume is the system volume except for the water/vacuum system where it is only the water volume. The solid lines are calculated numerically from Eqn. 17 and the other lines are calculated from Eqn. 24.

systems are difficult to compress this only corresponds to $2 \%$ difference in volume. Generally for the corrections to the energy (Eqn. 17) and pressures the surface correction is at least one, but usually two, orders of magnitude smaller than the bulk correction.

\section{CONCLUSION}

The surface tension at planar surfaces have contributions from weak long-range dispersion forces which in many cases are non-negligible for distances up to a few nanometers. For uniform systems, the contributions from long-range forces outside the cutoff $\left(r_{c}\right)$ to pressure and energy decay to zero as $r_{c}^{-3}$. For non-uniform systems, the leading contributions to the pressure components scales as $r_{c}^{-2}$ and are therefore more important. The average pressure is, however, not affected since the $r_{c}^{-2}$-terms of the different pressure components cancel. The surface tension, which may be obtained as an integral of the difference between the normal and lateral pressure, will then contain a leading correction term from long-range dispersion that scales as $r_{c}^{-2}$.

The simple and safe, but often computationally expensive, way around this problem is 
to use large cutoffs or some variant of Ewald summation not only for electrostatics but also for the faster decaying dispersion forces. The alternative is to apply long-range corrections to the pressure components. This should work unless the cutoff is small enough to cause structural differences in the system. It also requires some simple stable geometry (e.g. a planar one) in which it is feasible to calculate the corrections with reasonable effort. In other more complex geometries, generalized coordinates perpendicular and parallel to the surface would have to be introduced and the surface tension would have to be calculated from a spatially varying pressure tensor. If the shape of the surface varies in time the problem will be even more complex.

The purpose of the present work has been to systematically calculate the corrections at a planar surface and compare to simulations using cutoffs of different lengths including infinite cutoff (LJ-PME). The corrections to the surface tension have been calculated in two ways. The first is by numerically calculating a two-dimensional integral over a function containing the spatially varying dispersion density. This function was determined from the number density of different atoms (in the simulation) and the attractive van der Waals parameters of these atoms. Another excellent way is to approximate the interface using a tanh-function. Although the integral is still not analytically solvable, it may be Taylor-expanded in the dimensionless parameter given by the width of the tanh-function divided by the cutoff. This gives a leading $r_{c}^{-2}$-term which is the well-known result for a Heaviside function interface. The higher terms in the expansion will be higher powers of $r_{c}^{-2}$. The expansion will converge rather fast and it is not necessary to include higher terms than $r_{c}^{-4}$. The soft interface reduces the surface tension correction by less than 10-20\% compared to the sharp interface. The simulations show that the surface often is well represented by a tanh-function. Even when this is not that accurate (as for the lipid membrane), a comparison with numerically calculated corrections show that the resulting analytical corrections are still acceptable.

The main result of simulating four different systems (water, cyclohexane, cyclohexane/water and a lipid membrane) is that the surface tension scales to a good approximation 
as $\gamma=\gamma_{\infty}+a / r_{c}^{2}$ in all cases. A fourth order term can be determined but this is small. That $\gamma_{\infty}$ differs by $5-10 \%$ from experimental surface tensions just reflects lack of accuracy of the force fields used in the simulations. In principle, we would like to be able to predict the constants in front of the $r_{c}^{-2}$ - and $r_{c}^{-4}$-terms from the change in dispersion density at the surface and the width of the surface. This works to some extent.

For the membrane surface, the slope of the surface tension versus $r_{c}^{-2}$ is almost twice of what we anticipated from theory, although a fit could be obtained with the theoretical slope for cutoffs larger than $2 \mathrm{~nm}$. This indicates that the short cutoffs cause structural changes in the membrane that affect the surface tension. These are, however, more subtle than for the cyclohexane surface. For the water interface the agreement is excellent, while the agreement is reasonable for the water/cyclohexane interface. In the case of a pure cyclohexane interface, we note fairly large deviations at the shortest cutoffs. This indicates that the weakening of the cohesive interactions of the fluid due to the short cutoff brings us closer to the critical point and we also see a reduced number density of the fluid at these cutoffs.

\section{ACKNOWLEDGEMENTS}

This work is supported by SNIC (Swedish National Infrastructure for Computing) and the Parallel Computing Center at KTH (PDC).

\section{References}

(1) Ewald, P. P. Die Berechnung optischer und elektrostatischer Gitterpotentiale. Ann. Phys. 1921, 369, 253-287.

(2) Darden, T.; York, D.; Pedersen, L. Particle Mesh Ewald: An N.log(N) Method for Ewald Sums in Large Systems. J. Chem. Phys 1993, 98, 10089-10092.

(3) Wennberg, C. L.; Murtola, T.; Hess, B.; Lindahl, E. Lennard-Jones Lattice Summation 
in Bilayer Simulations Has Critical Effects on Surface Tension and Lipid Properties. $J$. Chem. Theory Comput. 2013, 9, 3527-3537.

(4) Kirkwood, J.; Buff, F. P. The Statistical Mechanical Theory of Surface Tension. J. Chem. Phys 1949, 17, 338-343.

(5) Chapela, G. A.; Saville, G.; Thompson, S. M.; Rowlinson, J. S. Computer Simulation of a Gas-liquid Surface. Part 1. J. Chem. Soc., Faraday Trans. 2 1977, 73, 1133-1144.

(6) Blokhuis, E.; Bedeaux, D.; Holcomb, C.; Zollweg, J. Tail Corrections to the Surface Tension of a Lennard-Jones Liquid-vapour Interface. Mol. Phys. 1995, 85, 665-669.

(7) Neyt, J.-C.; Wender, A.; Lachet, V.; Ghoufi, A.; Malfreyt, P. Quantitative Predictions of the Interfacial Tensions of Liquid-Liquid Interfaces through Atomistic and Coarse Grained Models. J. Chem. Theory Comput. 2014, 10, 1887-1899.

(8) Allen, M. P.; Tildesley, D. J. Computer Simulation of Liquids; Clarendon Press: New York, NY, USA, 1989.

(9) Safran, S. A. Statistical Thermodynamics of Surfaces, Interfaces, and Membranes; Addison-Wesley: Boulder, CO, USA, 1994.

(10) Lindahl, E.; Hess, B.; van der Spoel, D. GROMACS 3.0: a Package for Molecular Simulation and Trajectory Analysis. J. Mol. Model. 2001, 7, 306-317.

(11) van der Spoel, D.; Lindahl, E.; Hess, B.; Groenhof, G.; Mark, A. E.; Berendsen, H. J. C. GROMACS: Fast, Flexible, and Free. J. Comput. Chem. 2005, 26, 1701-1718.

(12) Pronk, S.; Páll, S.; Schulz, R.; Larsson, P.; Bjelkmar, P.; Apostolov, R.; Shirts, M. R.; Smith, J. C.; Kasson, P. M.; van der Spoel, D.; Hess, B.; Lindahl, E. GROMACS 4.5: a High-throughput and Highly Parallel Open Source Molecular Simulation Toolkit. Bioinformatics 2013, 29, 845-854. 
(13) Abascal, J. L. F.; Vega, C. A General Purpose Model for the Condensed Phases of Water: TIP4P/2005. J. Chem. Phys 2005, 123, 234505.

(14) Tjörnhammar, R.; Edholm, O. Reparameterized United Atom Model for Molecular Dynamics Simulations of Gel and Fluid Phosphatidylcholine Bilayers. J. Chem. Theory Comput. 2014, 10, 5706-5715.

(15) Haynes, W. M., Ed. CRC Handbook of Chemistry and Physics, 94th ed.; CRC Press, 2013.

(16) Parrinello, M.; Rahman, A. Polymorphic Transitions in Single Crystals: A New Molecular Dynamics Method. J. Appl. Phys. 1981, 52, 7182-7190.

(17) Bussi, G.; Donadio, D.; Parrinello, M. Canonical Sampling Through Velocity Rescaling. J. Chem. Phys 2007, 126, 014101.

(18) Lam, V. T.; Benson, G. C. Surface Tensions of Binary Liquid Systems. I. Mixtures of Nonelectrolytes. Can. J. Chem. 1970, 48, 3773-3781.

(19) Nijmeijer, M. J. P.; Bakker, A. F.; Bruin, C.; Sikkenk, J. H. A Molecular Dynamics Simulation of the Lennard-Jones Liquid-vapor Interface. J. Chem. Phys 1988, 89, 3789-3792.

(20) Stansfield, D. The Surface Tensions of Liquid Argon and Nitrogen. Proc. Phys. Soc. 1958, 72, 854-866.

(21) Vargaftik, N. B.; Volkov, B. N.; Voljak, L. D. International Tables of the Surface Tension of Water. J. Phys. Chem. Ref. Data 1983, 12, 817-820.

(22) Vega, C.; de Miguel, E. Surface Tension of the Most Popular Models of Water by Using the Test-area Simulation Method. J. Chem. Phys 2007, 126, 154707.

(23) Rehfeld, S. J. Adsorption of Sodium Dodecyl Sulfate at Various Hydrocarbon-Water Interfaces. J. Chem. Phys 1966, 71, 738-745. 
(24) Waheed, Q.; Edholm, O. Undulation Contributions to the Area Compressibility in Lipid Bilayer Simulations. Biophys. J. 2009, 97, 2754-2760.

(25) Kučerka, N.; Nagle, J. F.; Sachs, J. N.; Feller, S. E.; Pencer, J.; Jackson, A.; Katsaras, J. Lipid Bilayer Structure Determined by the Simultaneous Analysis of Neutron and XRay Scattering Data. Biophys. J. 2008, 95, 2356-2367. 


\section{Graphical TOC Entry}

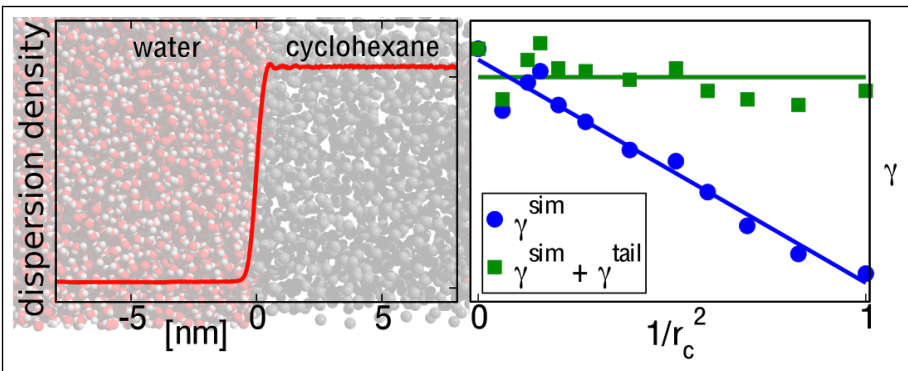

